\title{
Genome profiling revealed the activation of IL2RG/JAK3/STAT5 in peripheral T-cell lymphoma expressing the ITK-SYK fusion gene
}

\author{
LEI-LEI ZHANG ${ }^{1}$, HUA-XIONG PAN ${ }^{2}$, YI-XUAN WANG ${ }^{3}$, TAO GUO $^{1,4}$ and LIN LIU ${ }^{1}$ \\ Departments of ${ }^{1}$ Haematology, ${ }^{2}$ Pathology, ${ }^{3}$ Cardiovascular Surgery, Union Hospital, \\ Tongji Medical College, Huazhong University of Science and Technology; ${ }^{4}$ Collaborative Innovation Center of Haematology, \\ Huazhong University of Science and Technology, Wuhan, Hubei 430022, P.R. China
}

Received October 26, 2018; Accepted June 11, 2019

DOI: $10.3892 /$ ijo.2019.4882

\begin{abstract}
Peripheral T-cell lymphomas (PTCLs) are heterogeneous malignancies that are types of non-Hodgkin lymphomas; patients with this disease have poor prognoses. The IL-2-inducible T-cell kinase-spleen tyrosine kinase (ITK-SYK) fusion gene, the first recurrent chromosome translocation in PTCL-not otherwise specified (NOS), can drive cellular transformation and the development of T-cell lymphoma in mouse models. The aim of the current study was to investigate the signal transduction pathways downstream of ITK-SYK. The authors constructed a lentiviral vector to overexpress the ITK-SYK fusion gene in Jurkat cells. By using Signal-Net and cluster analyses of microarray data, the authors identified the tyrosine-protein kinase JAK (JAK)3/STAT5 signalling pathway as a downstream pathway of ITK-SYK, activation of which mediates the effects of ITK-SYK on tumourigenesis. JAK3-selective inhibitor tofacitinib abrogated the phosphorylation of downstream signalling molecule STAT5, supressed cell growth, induced cell apoptosis and arrested the cell cycle at the G1/S phase in ITK-SYK ${ }^{+}$Jurkat cells. In a xenograft mouse model, tumour growth was significantly delayed by
\end{abstract}

Correspondence to: Dr Tao Guo or Dr Lin Liu, Department of Haematology, Union Hospital, Tongji Medical College, Huazhong University of Science and Technology, Wuhan, Hubei 430022, P.R. China

E-mail: guotao1968@163.com

E-mail: doctorliulin@hotmail.com

Abbreviations: PTCLs, peripheral T-cell lymphomas; ITK-SYK, IL-2-inducible T-cell kinase-spleen tyrosine kinase; PTCL-NOS, peripheral T-cell lymphoma (not otherwise specified); PTCL-F, follicular variant of PTCL-NOS; MOI, multiplicity of infection; KEGG, Kyoto Encyclopedia of Genes and Genomes; IL2RG, interleukin-2 receptor subunit $\gamma$

Key words: peripheral T-cell lymphoma, IL-2-inducible T-cell kinase-spleen tyrosine kinase, JAK/STAT pathway, tofacitinib, interleukin-2 receptor subunit, xenograft mouse model tofacitinib. Since JAK3 associates with interleukin-2 receptor subunit $\gamma$ (IL2RG) only, siRNA-specific knockdown of IL2RG showed the same effect as tofacitinib treatment in vitro. These results first demonstrated that the activation of the IL2RG/JAK3/STAT5 signalling pathway contributed greatly to the oncogenic progress regulated by ITK-SYK, supporting further investigation of JAK3 inhibitors for the treatment of PTCLs carrying the ITK-SYK fusion gene.

\section{Introduction}

Peripheral T-cell lymphomas (PTCLs) are heterogeneous malignancies characterized by an aggressive clinical course and a poor outcome with current treatment strategies (1). The pathological diagnosis of PTCLs is often challenging due to its broad morphologic and immunophenotypic variabilities, and the occasional overlapping of distinct entities (2). The majority of PTCLs lack entity-defining genetic alterations. Therefore, they are named according to their predominant site of involvement, such as leukaemic, extranodal, cutaneous or nodal entities (3). The recent genome-wide molecular characterization of several entities has provided novel insights into their pathobiology and has led to the identification of new biomarkers with diagnostic, prognostic or therapeutic implications for patients with PTCL (4). Moreover, previously unrecognized recurrent genetic alterations have been described (5).

The $\mathrm{t}(5 ; 9)(\mathrm{q} 33 ; \mathrm{q} 22)$ translocation was first reported in 2006 in a small subset of PTCL-not otherwise specified (NOS) cases (6). The IL-2-inducible T-cell kinase (ITK) gene on chromosome 5 was translocated to the spleen tyrosine kinase (SYK) gene on chromosome 9 (7). The ITK-SYK translocation was predominantly detected in the follicular variant of PTCL-NOS (PTCL-F) (8). Soon thereafter, this translocation was also reported in angioimmunoblastic T-cell lymphoma (AITL) cases (9). Although clinical, morphologic and biological features distinguish PTCL-F from AITL (3), genetic translocation may imply a new entity in PTCLs.

To the best of our knowledge, this translocation represents the first fusion protein consisting of two non-receptor tyrosine kinases with important functions in lymphocyte development 
and signal transduction (10). ITK plays a critical role in all stages of T-cell development and differentiation $(11,12)$. SYK is involved in B cell receptor pathway activation, and its expression is markedly reduced in normal peripheral $\mathrm{T}$ lymphocytes $(13,14)$. The ITK-SYK fusion protein comprises the N-terminal PH-domain of ITK and the tyrosine kinase domain of SYK (15). In vitro and in vivo studies have shown that the constitutively active tyrosine kinase function of ITK-SYK is a key oncogenic event in the pathogenesis of ITK-SYK-positive PTCLs (7,16-18). ITK-SYK modulates signalling pathways, including $\mathrm{T}$ cell receptor (TCR), PI3K-Akt and mitogen-activated protein kinase (MAPK) signalling pathways $(15,18)$. However, the global impact of constitutive ITK-SYK expression in lymphoma cells is unknown.

\section{Materials and methods}

Cell culture and reagents. The human T-cell acute lymphoblastic leukaemia (T-ALL) cell lines Jurkat, Clone E6-1 (cat. no. TIB-152) and CCRF-CEM (cat. no. CCL-119) were obtained from the American Type Culture Collection. The Burkitt Lymphoma cell lines Raji (cat. no. TCHu 44) was acquired from the Cell Type Culture Collection in the Institute of Biochemistry and Cell Biology of Chinese Academy of Sciences (Shanghai, China). All the cell lines were grown in RPMI-1640 medium supplemented with $10 \%$ foetal bovine serum (FBS; both Gibco; Thermo Fisher Scientific, Inc.), penicillin $(100 \mathrm{U} / \mathrm{ml})$, and streptomycin $(100 \mathrm{mg} / \mathrm{ml}$; both HyClone; GE Healthcare Life Sciences) at $37^{\circ} \mathrm{C}$, with a $5 \%$ volume fraction of $\mathrm{CO}_{2}$ and $30 \%$ saturated humidity. The tyrosine-protein kinase JAK (JAK)3 inhibitor tofacitinib (cat. no. S5001; Selleck Chemicals) was dissolved in DMSO.

Lentiviral vector construction and transduction. The human ITK-SYK fusion gene was cloned from ITK and SYK human cDNA. The 494-bp ITK fragment was amplified using the following primer sequences: Forward, 5'-ATGAACAAC TTTATCCTCCTGGAA-3' and reverse, 3'-CCTGTTGTC TTCAGGAGTAGGAGG-5'. The 991-bp SYK fragment was amplified using the following primer sequences: Forward, 5'-TCC TCC CCTGCCCAAGGGAACCGGCAA-3' and reverse, 3'-TTAGTTCACCACGTCATAGTAGTAATT-5'. The two genes were ligated by a fusion PCR system using the following primer sequences: Forward, 5'-GACAACAGG TCCTCCCCT-3' and reverse, 3'-AGGGGAGGACCTGTT GTC-5'. The $20 \mu 1$ PCR volume contained $10 \mu 1$ Phusion Green Hot Start II High-Fidelity PCR Master mix (cat. no. F566L; Thermo Fisher Scientific, Inc.), $100 \mathrm{ng}$ DNA and $0.4 \mu \mathrm{M}$ of each primer. The PCRs were run on a C-1000 Thermal Cycler (Bio-Rad Laboratories) using the following cycling conditions: An initial denaturation at $98^{\circ} \mathrm{C}$ for $30 \mathrm{sec}$, followed by 35 cycles of $10 \mathrm{sec}$ at $98^{\circ} \mathrm{C}, 45 \mathrm{sec}$ at $62^{\circ} \mathrm{C}$ and $45 \mathrm{sec}$ at $72^{\circ} \mathrm{C}$, and a final extension for $10 \mathrm{~min}$ at $72^{\circ} \mathrm{C}$. The fusion PCR products were verified by DNA sequencing and then subcloned into the lentiviral vector pUbi-MCS-3FLAG-SV40-EGFP (GV287; Shanghai GeneChem Co., Ltd.). The overexpression vector was sent for sequencing and designated GV287-ITK-SYK, and the control vector was named GV287-NC. Jurkat cells, at a density of $1 \times 10^{5}$ cells $/ \mathrm{ml}$, were infected with lentiviral vectors plus $10 \mu \mathrm{g} / \mathrm{ml}$ polybrene (Sigma-Aldrich; Merck KGaA) at an
MOI of 50:1 for $16 \mathrm{~h}$. CEM cells were transduced as previously described, but at an MOI of 100:1. The number of the cells and mass of the transfected vectors were calculated according to the experiments. For an MOI of 50:1 in six-well plates, cells were seeded at $2 \times 10^{5}$ cells/well and $1 \times 10^{7}$ TU lentivirus vector was added. For an MOI of 50:1 in 24-well plate, cells were seeded at $1 \times 10^{5}$ cells/well and $5 \times 10^{6} \mathrm{TU}$ lentivirus vector TU lentivirus vector was added. The cells were maintained at $37^{\circ} \mathrm{C}$, with a $5 \%$ volume fraction of $\mathrm{CO}_{2}$ and $30 \%$ saturated humidity. After $72 \mathrm{~h}$, stably transfected Jurkat cells were grown in 1640 medium supplemented with $2 \mathrm{~g} / \mathrm{ml}$ puromycin and $10 \%$ FBS for 2 weeks. Stably transfected CEM cells were screened with $0.5 \mathrm{~g} / \mathrm{ml}$ puromycin as described above.

Soft agar colony formation assay. ITK-SYK ${ }^{+}$and ITK-SYK Jurkat cells $\left(5 \times 10^{3} /\right.$ well) were seeded in six-well plate in $0.35 \%$ (w/v) low-melting temperature agar (Sigma-Aldrich; Merck $\mathrm{KGaA}$ ) over layers of $0.75 \%$ low-melting temperature agar. After 3 weeks, the colonies were stained with $1 \%$ crystal violet solution (Sigma-Aldrich; Merck KGaA) for $10 \mathrm{~min}$ at room temperature and scored by counting under a light microscope under low power magnification (x40).

Microarray analysis. Genome-wide expression profiling analysis was performed by Genminix Informatics Co., Ltd. using the Affymetrix Gene Chip Human Gene 1.0 ST Array (Affymetrix; Thermo Fisher Scientific, Inc.) to identify ITK-SYK target genes and analyse related signalling pathways. For each sample, three biological replicates were performed. If the mRNA expression level of a gene had a 1.5-fold change the gene was determined to be differentially expressed. Differentially expressed mRNA was based primarily on the Kyoto Encyclopedia of Genes and Genomes (KEGG) database (http://www.kegg.jp/kegg/) $(19,20)$, which was used to analyse the potential functions of cross-genes involved in the pathway. Gene Ontology (GO) (21) enrichment analysis of DEGs was conducted using the Database for Annotation, Visualization, and Integrated Discovery (DAVID) (22). A P-value cut-off of $<0.05$ was set as the screening condition. An mRNA-mRNA interaction network was constructed using Cytoscape v3.0 software (http://cytoscape.org/) (23). The matrix of gene expression values was visualized graphically.

Cell transfection with small-interfering (si)RNAs targeting $I L 2 R G$. ITK-SYK ${ }^{+}$Jurkat cells $\left[2 \times 10^{6}\right.$ cells in $100 \mu 1$ of siPORT buffer (Ambion; Thermo Fisher Scientific, Inc.)] were transfected with $100 \mathrm{nM}$ anti-IL2RG (siIL2RG) or scrambled (si-control) siRNA (both Thermo Fisher Scientific, Inc.) via electroporation at $200 \mathrm{~V}$, with two 1.5 -msec pulses in two separate 96-well electroporation plates (Bio-Rad Laboratories) for $20 \mathrm{~min}$ at room temperature. The following three specific siIL2RG were assessed: siIL2RG-1, sense: 5'-CCTGAAGAA CCTAGAGGATCTTGTT-3', anti-sense: 5'-CCTAAGAAT CCGGAGTCTATAGGTT-3'; silL2RG-2, sense: 5'-CATTGG AGTGAATGGAGCCACCCAA-3'; anti-sense: 5'-CATTGA GTAAGGAGGCACCCGTCAA-3'; siIL2RG-3, sense: 5'-GGA CCACAGCTGGACTGAACAATCA-3', anti-sense: 5'-GGA GACAGGTCGTCAACAAACCTCA-3' and si-control, sense: 5'-GGACACGAATGCACTCAGATAGGTC-3'; anti-sense: 5'-AGACCGACGGTCGCGAGATATAATC-3'. Cells were 
used for experiments 2 days after transfection. Cells transfected with siRNAs were seeded in 24-well plates at a density of $2 \times 10^{5}$ cells/well and cell numbers were assayed by trypan blue exclusion daily for 4 days using Countess ${ }^{\mathrm{TM}}$ Automated Cell Counter (Invitrogen; Thermo Fisher Scientific, Inc.) $(24,25)$. Flow cytometric analysis was used to determine apoptosis and cell cycle distribution in transfected cells 3 days after transfection.

Western blotting. ITK-SYK ${ }^{+}$Jurkat cells were seeded at a density of $6 \times 10^{5}$ cells per well in six-well plates and further incubation with $0-5 \mu \mathrm{M}$ tofacitinib $(0,0.5,2.5$ and $5 \mu \mathrm{M})$ for $24 \mathrm{~h}$ at $37^{\circ} \mathrm{C}$. ITK-SYK ${ }^{+}$and siIL2RG-transfected ITK-SYK ${ }^{+}$ Jurkat cells were incubated with $25 \mathrm{ng} / \mathrm{ml} \mathrm{IL-2} \mathrm{(cat.} \mathrm{no.} \mathrm{202-IL)}$ or IL-21 (cat. no. 8879-IL; both R\&D Systems) for $24 \mathrm{~h}$ at $37^{\circ} \mathrm{C}$. Jurkat, Raji, ITK-SYK ${ }^{+}$or ITK-SYK Jurkat cells were seeded at a density of $6 \times 10^{5}$ cells per well in six-well plates. Wild-type SYK 72-kDa protein, expressed in Raji cells, was used as a comparison with the fusion SYK $55-\mathrm{kDa}$ protein. The cells were then harvested and washed twice with cold PBS. For whole-cell protein analysis, washed cells were lysed with cell lysis buffer radioimmunoprecipitation lysis buffer with protease and phosphatase inhibitor cocktail (both Beyotime Institute of Biotechnology). Total protein was measured using a BCA Protein assay kit (Beyotime Institute of Biotechnology). A total of $30 \mu \mathrm{g}$ protein per lane was separated by SDS-PAGE on $10 \%$ gels and then transferred onto polyvinylidene fluoride transfer membranes (EMD Millipore). The membranes were blocked by $5 \%$ bovine serum albumin (BSA; cat. no. A1933; Sigma-Aldrich; Merck KGaA) in TBST (0.1\% Tris-buffered saline with $1 \mathrm{ml} / 1$ Tween20) for $1 \mathrm{~h}$ at room temperature.

Then they were incubated overnight at $4^{\circ} \mathrm{C}$ with primary antibodies against SYK (cat. no. 13198), AKT (cat. no. 9272), phosphorylated (p-)AKT (cat. no. 9611), ERK (cat. no. 9102), p-ERK (cat. no. 4370), lymphocyte cytosolic protein 2 (SLP76; cat. no. 70896), p-SLP76 (cat. no. 92711), 1-phosphatidylinositol 4,5-bisphosphate phosphodiesterase $\gamma$-1 (PLCG1; cat. no. 5690), p-PLCG1 (cat. no. 8713), non-receptor tyrosine-protein kinase TYK2 (TYK2; cat. no. 14193), p-TYK2 (cat. no. 68790), JAK1 (cat. no. 3344), p-JAK1 (cat. no. 74129), JAK2 (cat. no. 3230), p-JAK2 (cat. no. 3776), JAK3 (cat. no. 8827), p-JAK3 (cat. no. 5031), STAT3 (cat. no. 12640), p-STAT3 (cat. no. 9145), STAT5 (cat. no. 25656), p-STAT5 (cat. no. 9314), caspase-3 (cat. no. 9665), cleaved caspase-3 (cat. no. 9664), cyclin-dependent kinase (CDK) inhibitor 1B (p27Kip1; cat. no. 3686), CDK2 (cat. no. 2546) and $\beta$-actin (cat. no. 4970; all 1:1,000; Cell Signalling Technology). Other primary antibodies used were the following: Interleukin-2 receptor subunit $\alpha$ (IL2RA; cat. no. ab128955), IL2R subunit $\beta$ (IL2RB; cat. no. ab61195), IL2R subunit $\gamma$ (IL2RG; cat. no. ab202911), IL7R (cat. no. ab155755) and IL21R (cat. no. ab5980; all 1:500; Abcam). After washing with TBST three times for $10 \mathrm{~min}$, the membranes were further incubated with horseradish peroxidase (HRP)-conjugated goat anti-rabbit immunoglobulin (Ig)G secondary antibodies (cat. no. ab6721; 1:4,000; Abcam) for $1 \mathrm{~h}$ at room temperature. The membrane was washed with TBST three times for $10 \mathrm{~min}$ and developed using SuperSignal West Pico Chemiluminescent Substrate (Thermo Fisher Scientific, Inc.) according to the protocol provided by the manufacturer's protocol. Densitometric analysis of the proteins band was performed by ImageJ software (version 1.6.0; National Institutes of Health).

$J A K 3$ inhibitor experiments in ITK-SYK $K^{+}$Jurkat cells. A total of $5 \times 10^{3}$ ITK-SYK $^{+}$or ITK-SYK- Jurkat cells per well were seeded in 96-well plate with RPMI-1640 medium containing DMSO, or $0.5,2.5$ or $5 \mu \mathrm{M}$ tofacitinib for $6,18,24$ and $48 \mathrm{~h}$ at $37^{\circ} \mathrm{C}$. DMSO exposure at a concentration of $0.1 \%$ in controls had no effect on the viability of examined cells lines, therefore $0.1 \%$ DMSO was used to treated the cells for $6,18,24$ and $48 \mathrm{~h}$ at $37^{\circ} \mathrm{C}$; these cells were used as the control cells. Cell viability was evaluated using a Cell Counting Kit-8 assay (CCK-8; Dojindo Molecular Technologies, Inc.) according to the manufacturer's protocol. Cells were measured at $450 \mathrm{~nm}$ using a Varioskan Flash multimode reader (Thermo Fisher Scientific, Inc.).

The apoptotic rate of transduced cells that were treated with tofacitinib was detected using an Annexin V-PE/7-ADD Apoptosis Detection kit (BD Pharmingen ${ }^{\mathrm{TM}}$ ). Separately, the transduced and tofacitinib-treated cells $\left(1 \times 10^{6}\right)$ were stained with propidium iodide (Sigma-Aldrich; Merck KGaA) at room temperature in the dark for $30 \mathrm{~min}$. The cells were analysed using a flow cytometer using ModFit LT version 5.0 analysis software (Verity Software House, Inc.). Experiments were performed in triplicate.

$J A K 3$ inhibitor in a xenograft mouse model. Female NOD/SCID mice with a median weight of 14-16 g $(n=14$; 4-6 weeks old; Huafukang Co., Ltd.) were housed in a dedicated pathogen-free barrier facility at the Laboratory Animal Center of Tongji Medical College (Wuhan, China). The mice were kept in separate cages in a room with specific pathogen-free standards at a temperature of $22 \pm 2^{\circ} \mathrm{C}$ and $50 \pm 10 \%$ relative humidity, with food and water available ad libitum. The animal room was on a 12/12-h light/dark cycle. The CEM cells were transduced with GV287-ITK-SYK at an MOI of 100:1 as described above. After 7 days, 5x10 transduced CEM cells suspended in a volume of $50 \mu 1$ RPMI-1640 medium were mixed with $50 \mu 1$ Matrigel (BD Biosciences) and subcutaneously inoculated into the rear flank of NOD/SCID female mice. Tumour-bearing mice (average tumour volume, $100 \mathrm{~mm}^{3}$ ) were randomly divided into two groups: A tofacitinib group $(n=6)$ and a control group ( $\mathrm{n}=6)$. Tofacitinib (20 mg/kg per day) was administered via oral gavage for 28 consecutive days and $100 \mu 11 \mathrm{X}$ PBS was used as the vehicle. Tumour volumes were monitored in 3-day intervals with electronic callipers and were calculated as follows: Volume $\left(\mathrm{mm}^{3}\right)=$ length $\mathrm{x}$ width $\mathrm{x}$ thickness. Mice were depilated once a week to reduce the background fluorescence of mouse hair under short-wavelength excitation light. Fluorescence imaging was performed at the end of the experiment using an in vivo imaging system Fx Pro (Bruker Corporation) under $488 \mathrm{~nm}$ excitation and $510 \mathrm{~nm}$ emission for green fluorescence. Fluorescent intensity was visualized by enhanced green fluorescent protein (EGFP) in NOD/SCID mice. The intensity of the region of interest (ROI) was plotted in units of maximum number of photons per second per centimetres squared per steradian ( $\left.\mathrm{p} / \mathrm{sec} / \mathrm{cm}^{2} / \mathrm{sr}\right)$, ROIs were drawn over the signals and average radiant efficiency was quantified 
in terms of $\mathrm{p} / \mathrm{s} / \mathrm{cm}^{2} / \mathrm{sr}$. All mice were sacrificed by $\mathrm{CO}_{2}$ inhalation (flow rate, $20 \% \mathrm{CO}_{2} / \mathrm{min}$ ) (26) at 28 days after the start of tofacitinib treatment and tumours were removed.

Tumour tissues were fixed with $10 \%$ formaldehyde solution overnight at room temperature and then embedded in paraffin, the tumours were cut into serial sections $\sim 2-3 \mu \mathrm{m}$ thick. Sections were subjected to haematoxylin and eosin (both Sigma-Aldrich; Merck KGaA) staining for 10 and $5 \mathrm{~min}$, respectively, at room temperature) and visualized under high power magnification (x200) on a light microscope. For immunohistochemical staining assay, paraffin-embedded sections were deparaffinized in xylene, rehydrated in a graded series of alcohols (100, 95 and 75\%) and rinsed twice with PBS. Antigen retrieval was performed at $60^{\circ} \mathrm{C}$ for $2 \mathrm{~h}$, then the samples were dewaxed in xylene for 10 min twice. Samples were then dehydrated in anhydrous ethanol for $5 \mathrm{~min}$ twice, $95 \%$ ethanol for $2 \mathrm{~min}, 90 \%$ ethanol for $2 \mathrm{~min}, 80 \%$ ethanol for $2 \mathrm{~min}, 70 \%$ ethanol for $2 \mathrm{~min}$. Following antigen retrieval, slides were incubated with $3 \%$ $\mathrm{H}_{2} \mathrm{O}_{2}$ for $30 \mathrm{~min}$ to inhibit endogenous peroxidase. Slides were then blocked with $5 \% \mathrm{BSA}$ at $20^{\circ} \mathrm{C}$ for $2 \mathrm{~h}$ and incubated with primary antibodies against SYK (cat. no. sc1240) at $4^{\circ} \mathrm{C}$ overnight; the antibodies were purchased from Santa Cruz Biotechnology, Inc. and diluted to 1:100. After washing in PBS, the tissue sections were treated with HRP-conjugated goat anti-mouse IgG (cat. no. A4416; 1:1,000; Sigma-Aldrich; Merck KGaA) for $1 \mathrm{~h}$ at room temperature. Subsequently, $0.1 \%$ diaminobenzidine (Sigma-Aldrich; Merck KGaA) was used as a chromogen at $25^{\circ} \mathrm{C}$ for $1 \mathrm{~min}$ and $10 \%$ haematoxylin was used to re-dye the sections at $25^{\circ} \mathrm{C}$ for $3 \mathrm{~min}$. The images were viewed under high power magnification (x400) on a light microscope. A semi-quantitative analysis of JAK3 expression in stained sections was assessed by an independent pathologist. The German semi-quantitative scoring system was applies to score the intensity and extent of staining. Immunohistochemical staining intensity scores were determined by multiplying the staining intensity of SYK-positive cells $(0=$ negative, $1=$ weak, $2=$ moderate and $3=$ strong) with the extent of positively stained cells $(4=75-100 \%, 3=50-74 \%, 2=25-49 \%, 1=1-24 \%$ and $0=0 \%)$, leading to scores from 0 to 12 . The study was approved on $01 / 16 / 2017$ by the Institutional Animal Care and Use Committee at Tongji Medical College, Huazhong University of Science and Technology (Wuhan, China; ACUC no. S842).

ELISA. IL-2 was measured in the supernatants of untransfected Jurkat cells incubated with $10 \mu \mathrm{g} / \mathrm{ml}$ anti-CD28 and anti-CD3 (clone UCHT-1; both BD Pharmingen; BD Biosciences) at $37^{\circ} \mathrm{C}$ for $72 \mathrm{~h}$, ITK-SYK ${ }^{+}$Jurkat cells, ITK-SYK- Jurkat cells. All ILs were measured in the supernatants of ITK-SYK- Jurkat cells, ITK-SYK ${ }^{+}$Jurkat cells and ITK-SYK ${ }^{+}$Jurkat cells transfected with si-control or si-IL2RG. All cells were seeded in a six-well plate at a density of $2 \times 10^{5}$ cells/well and incubated for $72 \mathrm{~h}$ at $37^{\circ} \mathrm{C}$, with a $5 \%$ volume fraction of $\mathrm{CO}_{2}$ and $30 \%$ saturated humidity. IL-2 (cat. no. D2050), IL-4 (cat. no. D4050), IL-7 (cat. no. HS750), IL-9 (cat. no. DY209-05), IL-15 (cat. no. D1500) and IL-21 (cat. no. DY8879-05) protein concentrations were measured in triplicate using ELISA kits (R\&D Systems) according to the manufacturer's protocols.
Statistical analysis. Each experiment was performed independently three times; the results of the independent experiments were similar. The results were expressed as the mean \pm SD. Significant differences between two groups were assessed with Student's t-test (parametric) and Mann-Whitney U test (non-parametric). The significance among multiple groups was using one-way ANOVA followed by Dunnett's post-hoc test. All analyses were performed with GraphPad Prism6 Software (GraphPad Software, Inc.). $\mathrm{P}<0.05$ was considered statistically significant.

\section{Results}

An ITK-SYK fusion construct is overexpressed in cell lines. To study the role of ITK-SYK in lymphomagenesis, a lentiviral vector carrying the ITK-SYK fusion gene was constructed as previously described (2). First, the fusion construct containing ITK and SYK was engineered and a sequence analysis of the 2.4-kb PCR product was performed to confirm the fusion of N-terminal ITK to C-terminal SYK (Fig. S1A). Next, the authors assessed whether the functional expression of the ITK-SYK fusion construct in mammalian cell lines was feasible. The fusion gene was subcloned into lentiviral vectors and Jurkat cells were then transduced the lentiviral vectors. ITK-SYK ${ }^{+}$Jurkat cells were detected under a fluorescence microscope to observe GFP expression (Fig. S1B) and the transduction efficiency exceeded $85.23 \%$ as observed by flow cytometry (Fig. S1C). Transduction of Jurkat cells with the fusion construct resulted in the expression of a stable $55-\mathrm{kDa}$ protein corresponding to full-length ITK-SYK, which was shorter than the wild-type SYK 72-kDa protein expressed in Raji cells; Jurkat cells transduced with control vectors did not express SYK protein (Fig. 1B). Collectively, these results showed that the ITK-SYK fusion gene was successfully expressed in cells.

Genome-wide expression profiling of ITK-SYK-transduced cells. As previously reported (27), ITK-SYK ${ }^{+}$Jurkat cells exhibited significantly increased colony-forming ability, significantly greater IL-2 secretion and oncogenic signalling compared with ITK-SYK- Jurkat cells (Fig. 1A, C and E). To further understand the molecular mechanism underlying ITK-SYK function, mRNA expression profiling was performed to identify the genes influenced by ITK-SYK. A total of 722 differentially expressed genes (DEGs), including 278 upregulated and 444 downregulated genes, were identified in ITK-SYK ${ }^{+}$Jurkat cells ( $\mathrm{P}<0.05$; fold change $>1.5$; Fig. 1D). Next, gene set enrichment analysis was performed to further define the biological processes altered by ITK-SYK. Gene Ontology terms of DEGs in ITK-SYK ${ }^{+}$Jurkat cells were enriched, such as cell cycle, apoptosis, DNA repair, signal transduction and cell proliferation (Fig. 1F). Additionally, KEGG pathway that were enriched included paths in cancer, such as the PI3K/Akt signalling pathway, the MAPK signalling pathway and the JAK-STAT signalling pathway (Fig. 1G).

ITK-SYK transduction activates the JAK3/STAT5 signalling pathway. To gain insight into gene interactions for the discovery of a novel candidate in ITK-SYK-mediated tumourigenesis, a Signal-Net analysis was performed to 
A

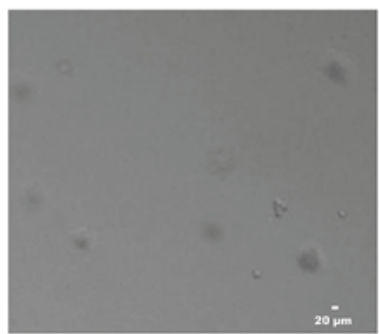

GV287-NC

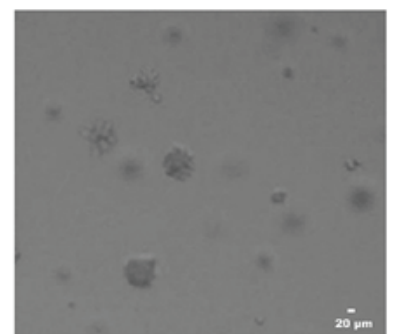

GV287-ITK-SYK

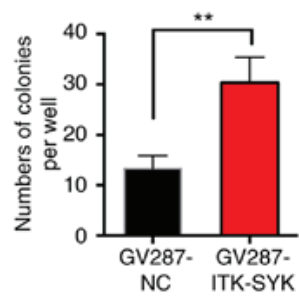

B
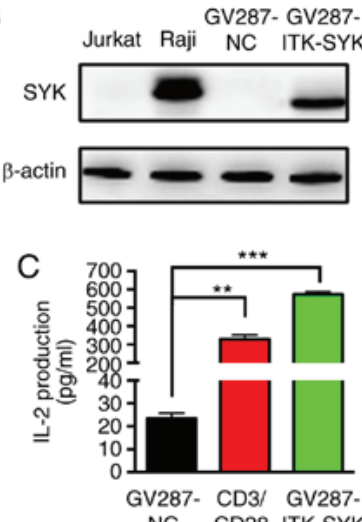

D

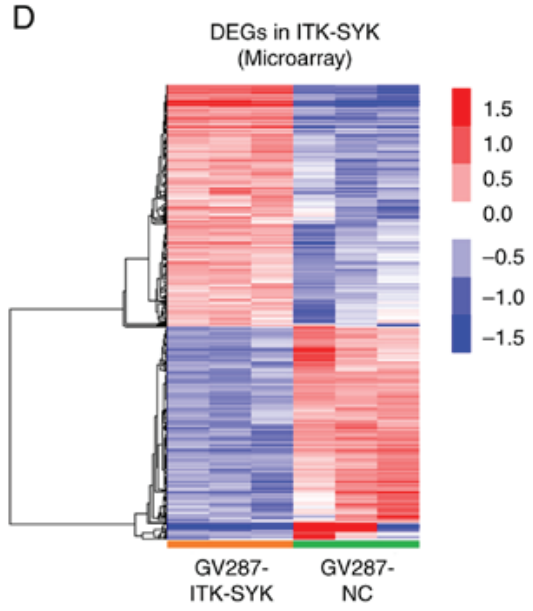

$\mathrm{E}$

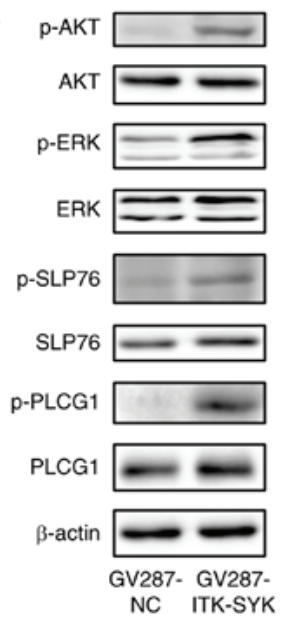

F

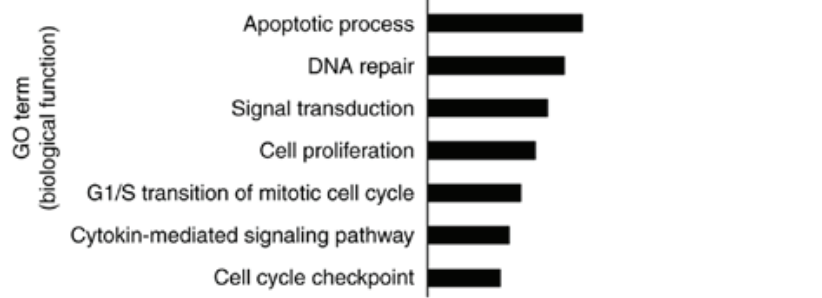

G

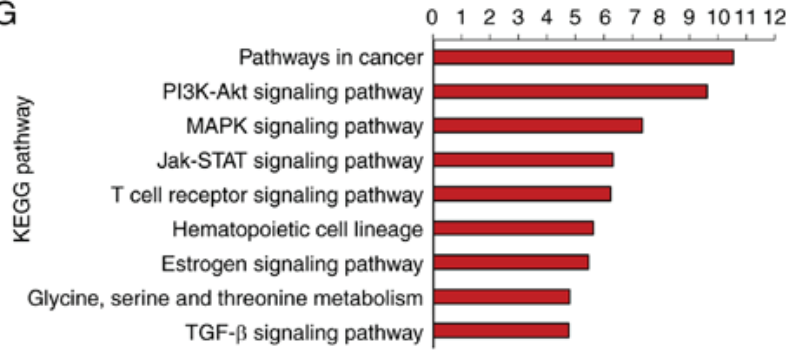

Figure 1. Genome profiling of DEGs mediated by the ITK-SYK fusion gene. (A) Jurkat cells at a density of $10^{5}$ cells per well were stably transfected with a GV287-NC or GV287-ITK-SYK vector in six-well plates in a soft agar colony formation assay. Colonies were allowed to form for 21 days. The colonies were then photographed in microscopic fields, as shown in the left panels; quantification of macroscopically visible colonies is shown in the right panel. Scale $\mathrm{ba}=20 \mu \mathrm{m}$. Data are expressed as the mean \pm SD from triplicate samples. (B) Cell lysates of Jurkat, Raji, and Jurkat cells transfected with GV287-NC or GV287-ITK-SYK were evaluated with western blotting analysis for the expression levels of SYK. $\beta$-actin was used as the loading control. (C) Jurkat cells were transfected with GV287-NC or GV287-ITK-SYK and stimulated with $10 \mu \mathrm{g} / \mathrm{ml}$ anti-CD3/CD28. IL-2 concentrations (pg/ml) in the cell supernatants were determined by ELISA. Data are expressed as the mean \pm SD from triplicate samples. (D) A heat map of DEGs in ITK-SYK-transfected Jurkat cells measured by mRNA microarray data. Upregulated genes were shown in red and downregulated genes in blue. (E) Total and phosphorylated levels of AKT, ERK, SLP76 and PLCG were measured by western blotting analysis. $\beta$-actin was used as the loading control. (F) GO term and (G) KEGG pathway enrichment analysis of DEGs in ITK-SYK-transduced Jurkat cells. ${ }^{* *} \mathrm{P}<0.01$ and ${ }^{* * * *} \mathrm{P}<0.001$. DEGs, differentially expressed genes; ITK-SYK, IL-2-inducible T-cell kinase-spleen tyrosine kinase; GO, Gene Ontology; KEGG, Kyoto Encyclopedia of Genes and Genomes; p-, phosphorylation; IL, interleukin; SLP76, lymphocyte cytosolic protein 2; PLCG1, 1-phosphatidylinositol 4,5-bisphosphate phosphodiesterase $\gamma$-1; NC, negative control; CD, cluster of differentiation; TGF, transforming growth factor.

generate a network based on the fold change level in microarray data. The top genes within the network were defined as key factors. The resulting network indicated that genes with the highest degrees of interaction were JAK3 and STATs (STAT3, STAT5A and STAT5B), indicating JAK/STAT pathway activation (Fig. 2A). To the best of our knowledge, 

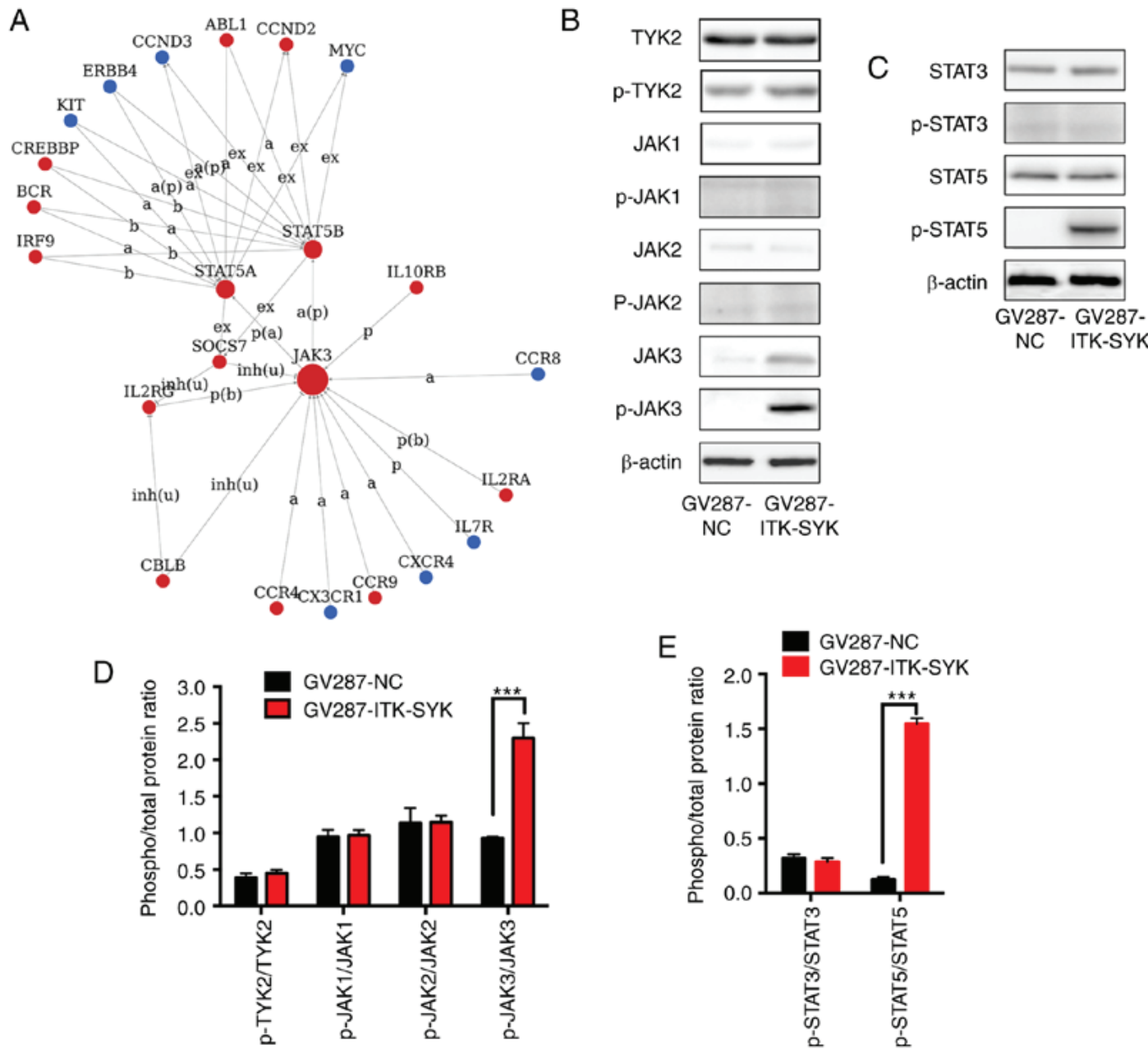

Figure 2. The JAK3/STAT5 signalling pathway is activated in ITK-SYK-transduced cells. Jurkat cells were transduced with GV287-ITK-SYK and GV287-NC. (A) Signal-Net analysis of top genes. The nodes in the figure represent the main genes, the edges represent interactions between genes and the arrows point toward the targets. The type of interaction between two genes is denoted by the letters and abbreviations on the edges. Red nodes represent upregulated genes, green nodes represent downregulated genes. The size of the circle was the frequency at which the gene interacts with other genes in the signalling network. The most frequent genes were the most prominent central genes in the network. (B) The expression levels of TYK2, JAKs and their phosphorylated counterparts were evaluated with western blotting analysis. (C) The expression levels of total and phosphorylated STAT3 and STAT5 were evaluated with western blotting analysis. $\beta$-actin was used as the loading control. Densitometric analysis of (D) total and phosphorylated TYK2 and JAKs, and (E) total and phosphorylated STAT3 and STAT5. Data is expressed as the mean \pm SEM of the phosphorylated/total protein ratio. Experiments were repeated at least three times. ${ }^{* * *} \mathrm{P}<0.001$. a, activation; b, binding; ex, expression; p, phosphorylation; inh(u), inhibition (ubiquitination); ITK-SYK, IL-2-inducible T-cell kinase-spleen tyrosine kinase; TYK2, non-receptor tyrosine-protein kinase; JAK, tyrosine-protein kinase JAK; NC, negative control.

the JAK/STAT signalling pathway in relation to ITK-SYK ${ }^{+}$ PTCLs has never been reported in previous studies; therefore, the authors of the current study set out to investigate the role of the JAK/STAT signalling pathway in ITK-SYK ${ }^{+}$PTCLs. Firstly, the expression of JAK1, JAK2, JAK3 and TYK2 was detected by western blotting, and it was found that JAK3 protein levels moderately increased in ITK-SYK ${ }^{+}$Jurkat cells, while JAK1, JAK2, and TYK2 protein levels were not influenced by ITK-SYK (Fig. 2B and D). The upregulation of JAK3 was consistent with the microarray data. Furthermore, JAK3 was constitutively activated by phosphorylation in ITK-SYK ${ }^{+}$ Jurkat cells; the ratio of p-JAK3/JAK3 was significantly higher in the ITK-SYK ${ }^{+}$Jurkat cells compared with the cells transduced with the control vector. Next the authors of the current study sought to identify the downstream signalling pathway activated by JAK3, and analysed the activity of STAT3 and STAT5 in ITK-SYK ${ }^{+}$Jurkat cells. JAK3 induced the constitutive phosphorylation of STAT5 in ITK-SYK ${ }^{+}$Jurkat cells, while the expression and phosphorylation of STAT3 were not influenced (Fig. 2C and E). The ratio of p-STAT5/STAT5 was significantly higher in the ITK-SYK ${ }^{+}$Jurkat cells compared with the cells transduced with the control vector.

JAK3 inhibition affects the survival of ITK-SYK $K^{+}$Jurkat cells. To determine the functional role of JAK3 in the survival of ITK-SYK ${ }^{+}$Jurkat cells, the effects of the target-selective small molecular inhibitor tofacitinib on ITK-SYK ${ }^{+}$Jurkat cells was evaluated. The results showed that tofacitinib obviously inhibited the survival of ITK-SYK ${ }^{+}$Jurkat cells compared with ITK-SYK- Jurkat cells. Furthermore, these inhibitory effects increased with tofacitinib concentration $(0.5,2.5$ and $5 \mu \mathrm{M})$ and treatment time $(6,18,24$ and $48 \mathrm{~h})$, indicating dose and time dependency of the inhibitory effects on cell viability on ITK-SYK ${ }^{+}$Jurkat cells $(50 \%$ reduction by $2.5 \mu \mathrm{M}$ at $48 \mathrm{~h}$; Fig. 3A and B). The inhibitory effects on ITK-SYK- Jurkat cells did not indicate dose and time dependency (Fig. 3B). Similarly, 

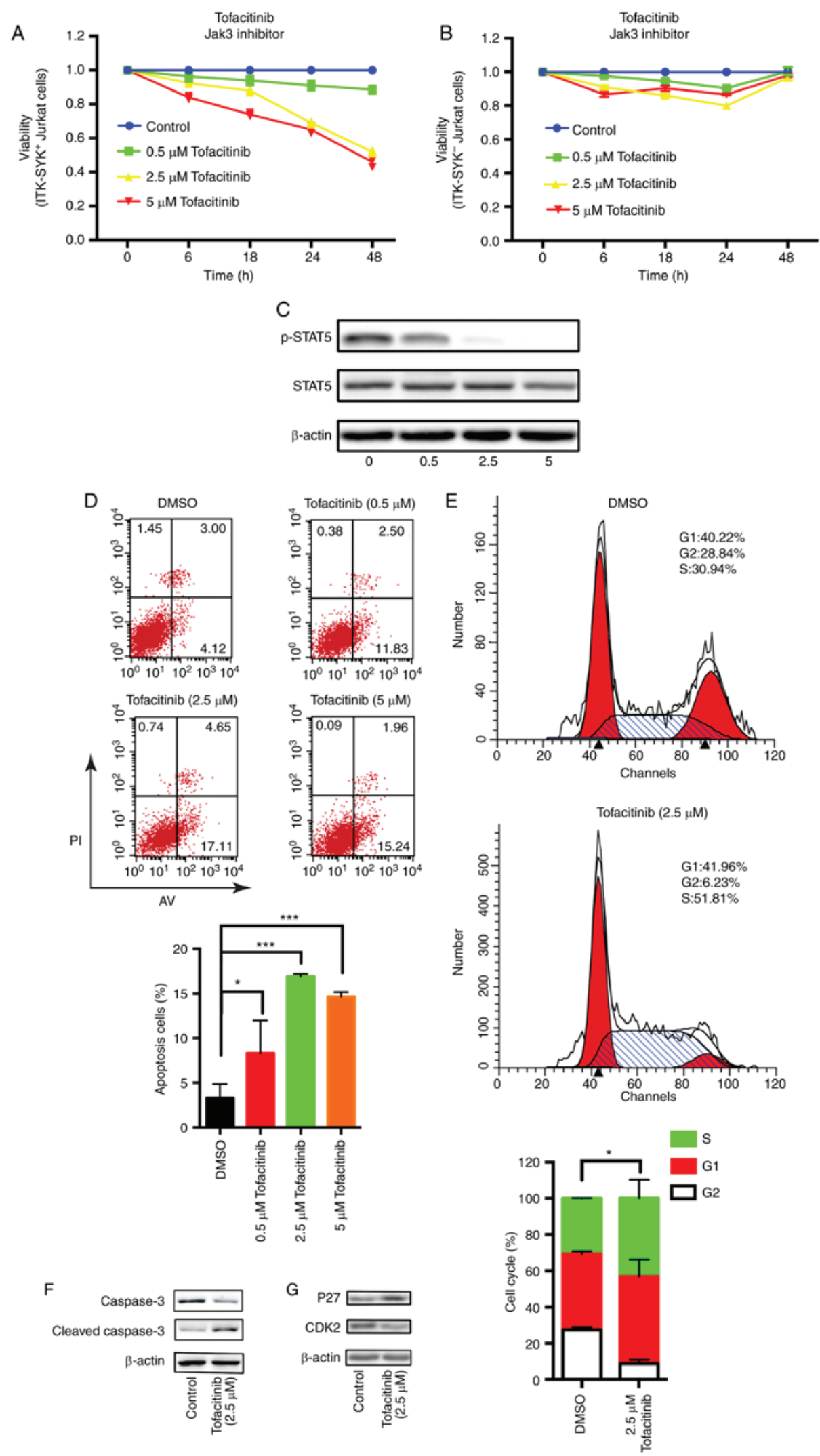

Figure 3. JAK3 inhibition affects ITK-SYK ${ }^{+}$Jurkat cells survival. Jurkat cells were transduced with GV287-ITK-SYK or GV287-NC for 72 h. (A) ITK-SYK and (B) ITK-SYK- Jurkat cells were incubated with $0.5,2.5$ and $5.0 \mu \mathrm{M}$ tofacitinib (a JAK3 inhibitor) or DMSO for up to 48 h. Cell viability was evaluated by the Cell Counting Kit- 8 assay. The data represent the mean percentage of viability relative to DMSO control obtained from experiments performed in triplicate. (C) The expression levels of total and phosphorylated STAT5 after $24 \mathrm{~h}$ of tofacitinib or DMSO treatment. (D) Flow cytometry analysis with Annexin V-PI staining was performed to evaluate the percentage of apoptotic cells; the results are quantified and showed as statistical analysis chart in the lower panel. The ITK-SYK ${ }^{+}$Jurkat cells were treated with $2.5 \mu \mathrm{M}$ tofacitinib or DMSO for $24 \mathrm{~h}(\mathrm{E})$, Cells were fixed and stained with PI. Cell cycle profiles were assessed using flow cytometry; the results are quantified and showed as statistical analysis chart in the lower panel. (F) Cleaved caspase-3 and full-length caspase-3, and (G) $\mathrm{p} 27$ and CDK2 expression levels were evaluated with western blotting analysis. $\beta$-actin was used as the loading control. ${ }^{*} \mathrm{P}<0.05,{ }^{* *} \mathrm{P}<0.01$ and ${ }^{* * * *} \mathrm{P}<0.001$. ITK-SYK, IL-2-inducible T-cell kinase-spleen tyrosine kinase; NC, negative control; PI, propidium iodide; AV, Annexin V; p27, cyclin-dependent kinase inhibitor 1B; CDK2, cyclin-dependent kinase 2. 

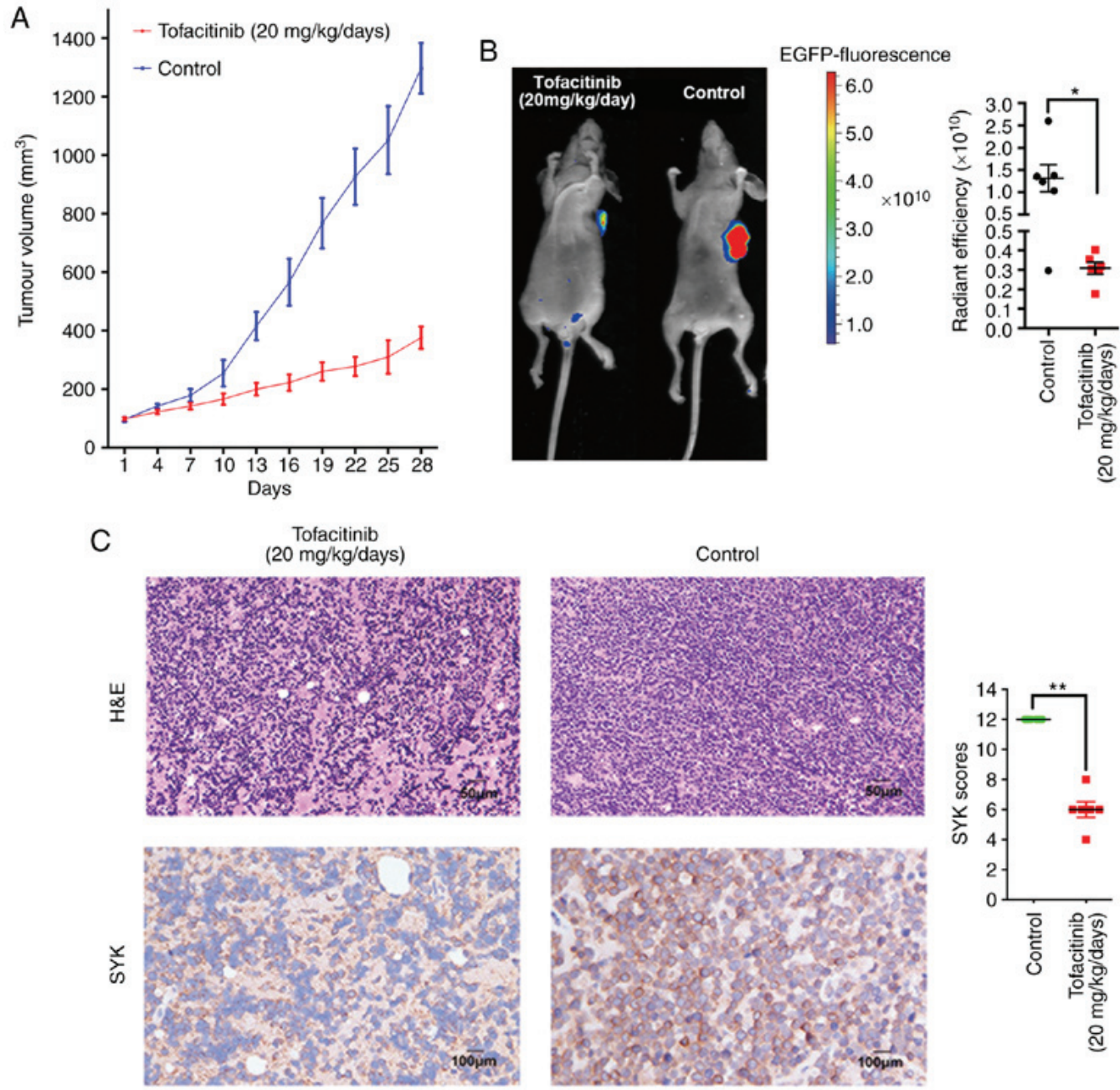

Figure 4. Tofacitinib inhibits the growth of an established tumour in a xenograft mouse model. ITK-SYK ${ }^{+}$CEM cells $\left(5 \times 10^{6}\right.$ cells per mouse) were mixed with Matrigel were subcutaneously inoculated into the flanks of mice. Xenograft mice were treated with tofacitinib ( $20 \mathrm{mg} / \mathrm{kg} / \mathrm{day})$ or equivalent PBS by oral gavage for 28 consecutive days. $n=6$ mice per group. (A) Tumour volume assessment following tofacitinib treatment. Tumour size was quantified in 3-day intervals. (B) Fluorescence images of tumour-bearing mice are shown after 4 weeks of treatment with tofacitinib or PBS; quantification of radiant efficiency is shown in the right panel. (C) H\&E and immunohistochemical staining in tumours obtained from the tofacitinib and control groups. Scale ba=50 $\mu \mathrm{m}$ (upper panel) and $100 \mu \mathrm{m}$ (lower panel). Immunohistochemical staining with anti-SYK antibodies was performed on the control and tofacitinib groups. Representative images are shown in the panels on the left and quantification is shown on the right. ${ }^{*} \mathrm{P}<0.05$ and ${ }^{* *} \mathrm{P}<0.01$. EGFP, enhanced green fluorescence protein; ITK-SYK, IL-2-inducible T-cell kinase-spleen tyrosine kinase; H\&E, haematoxylin and eosin.

ITK-SYK ${ }^{+}$Jurkat cells were treated with increasing concentrations of tofacitinib $(0.5,2.5$ and $5 \mu \mathrm{M})$ for $24 \mathrm{~h}$, which caused STAT5 phosphorylation to markedly decrease (Fig. 3C).

ITK-SYK ${ }^{+}$Jurkat cells treated with $2.5 \mu \mathrm{M}$ tofacitinib exhibited a significantly higher apoptosis rate compared with those treated with DMSO (tofacitinib $17.11 \%$ vs. control 4.12\%; $\mathrm{P}<0.001$; Fig. 3D). The apoptosis rate of cells treated with $0.5 \mu \mathrm{M}$ or $5 \mu \mathrm{M}$ tofacitinib increased compared with those treated with DMSO $(0.5 \mu \mathrm{M}$ tofacitinib $11.83 \%$ vs. control $4.12 \% ; 5 \mu \mathrm{M}$ tofacitinib $15.24 \%$ vs. control $4.12 \%$; $\mathrm{P}<0.05)$. However, increased concentrations of tofacitinib beyond $2.5 \mu \mathrm{M}$ did not lead to increases in the apoptosis rate. ITK-SYK ${ }^{+}$Jurkat cells were treated with $2.5 \mu \mathrm{M}$ tofacitinib or DMSO for $24 \mathrm{~h}$. Consistent with the results obtained by flow cytometry, increased levels of cleaved caspase-3, together with decreased levels of full-length caspase-3, were observed in the tofacitinib group (Fig. 3F). Cell cycle analysis demonstrated a significant increase in the number of tofacitinib-treated cells in the G1/S phase compared with the control group (control
$71.16 \%$ vs. tofacitinib $93.77 \%$; $\mathrm{P}<0.05$; Fig. 3E). Consistent with G1/S phase arrest, increased levels of p27 and decreased levels of CDK2 were observed in the tofacitinib group (Fig. 3F). The combined results suggested that JAK3 inhibitor tofacitinib affected cell proliferation, the cell cycle and apoptosis in ITK-SYK ${ }^{+}$Jurkat cells.

Tofacitinib inhibited the growth of established tumours in a xenograft mouse model. The current study was extended to an in vivo xenograft model to validate the significance of the in vitro findings. Cells from the T-ALL cell line CEM were transduced with lentiviral vectors and then used for the in vivo xenograft model as described in a previous study (28). The authors of the current study then subcutaneously inoculated $5 \times 10^{6}$ ITK-SYK ${ }^{+}$CEM cells into mice. Tofacitinib $(20 \mathrm{mg} / \mathrm{kg} / \mathrm{day})$ or equivalent PBS was administered with oral gavage for 28 consecutive days. Compared with control mice, tofacitinib-treated mice showed a marked delay in tumour growth at the end of the experiment (Fig. 4A). The 

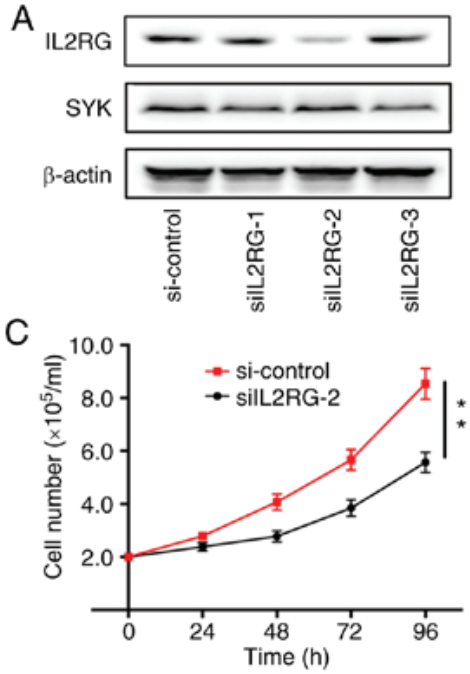

D

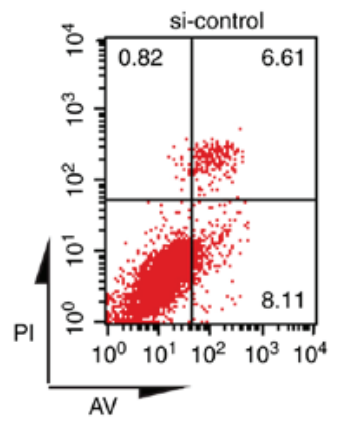

F

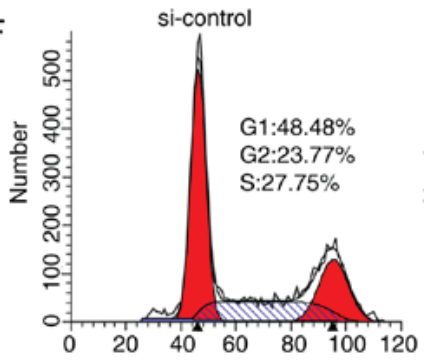

B

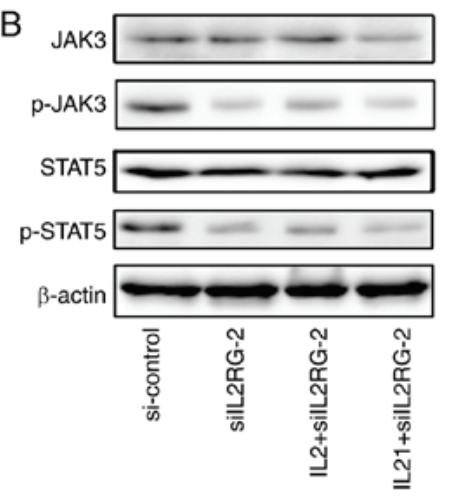

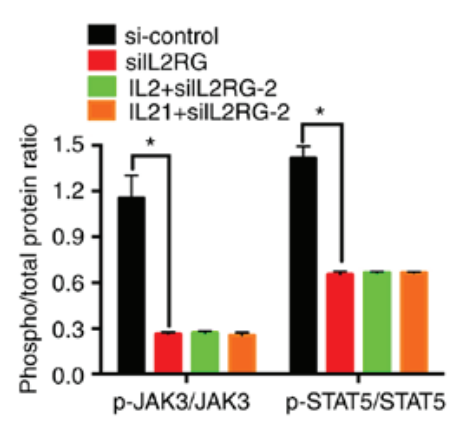

E

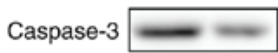

Cleaved caspase-3

$\beta$-actin

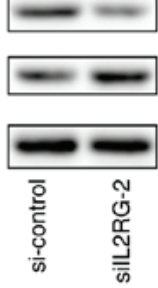

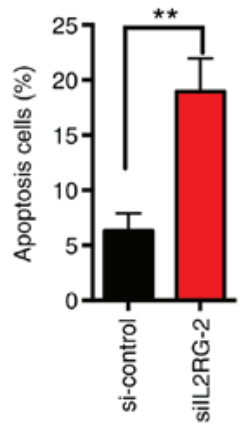

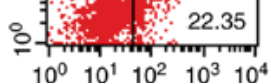
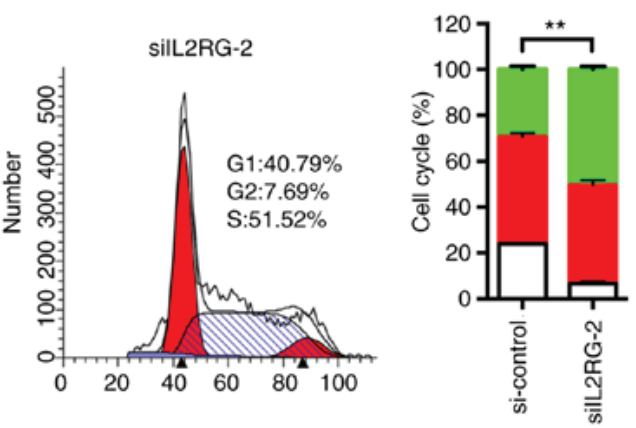

G
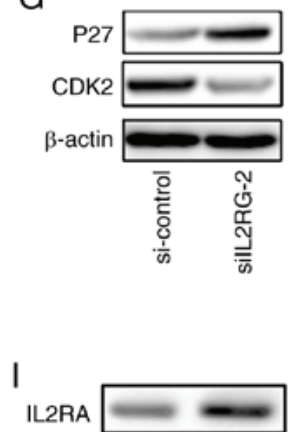

$\mathrm{H}$

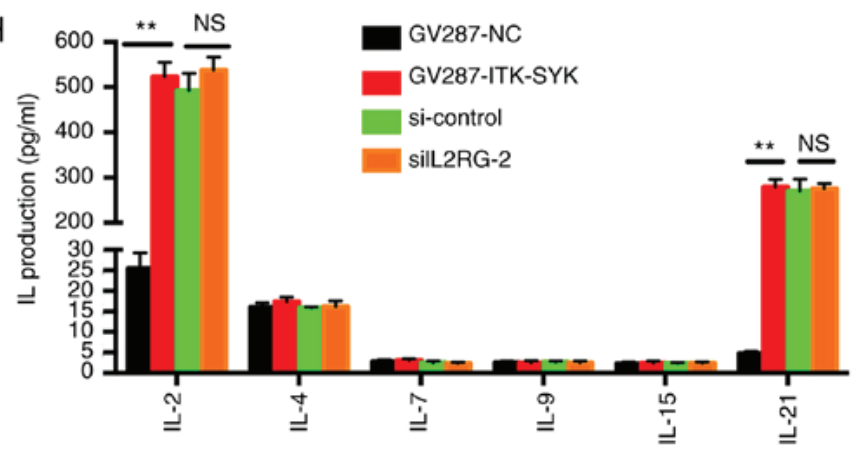

Figure 5. JAK3 phosphorylation is dependent on the expression of IL2RG, and associated with the secretion of IL-2 and IL-21. ITK-SYK ${ }^{+}$Jurkat cells were transfected with silL2RG or si-control for $48 \mathrm{~h}$. (A) Western blot analysis of IL2RG and SYK. (B) Exogenous IL-2 (25 ng/ml) or IL-21 (25 ng/ml) was added to the Jurkat cells for $24 \mathrm{~h}$. Cell lysates of si-control and IL2RG-knockdown Jurkat cells, and IL2RG-knockdown Jurkat cells treated with IL-2 or IL-21 were evaluated with western blotting analysis for the expression levels of JAK3, p-JAK3, STAT5 and p-STAT5. Densitometric analysis of the western blotting bands is shown in the graph on the right. Data is expressed as the mean $\pm \mathrm{SEM}$ of the phosphorylated/total protein ratio. Data is expressed as phospho/total protein ratio. (C) Growth curves in siIL2RG and si-control cells by cell counting. The results are expressed as the mean \pm SD of five independent experiments. (D) Flow cytometry analysis with Annexin V-PI staining was performed to evaluate the percentage of apoptotic cells between siIL2RG and si-control cells; the results are quantified in the graph on the right. (E-G) The transduced and transfected Jurkat cells were cultured for 24 h. (E) Cleaved caspase-3 and full-length caspase-3 were evaluated with western blotting analysis. (F) The cells were fixed and stained with PI. Cell cycle profiles were assessed using flow cytometry; the results are quantified in the right panel. (G) p27 and CDK2 expression levels were evaluated with western blotting analysis. (H) The protein concentrations of cytokines (IL-2, IL-4, IL-7, IL-9, IL-15, IL-21) were determined by ELISA ( $\mathrm{n}=3$ ). Quantification of the ELISAs is shown in the panel on the right. (I) Western blotting analysed the expression levels of IL2R, IL7R and IL21R. $\beta$-actin was used as the loading control. PI, propidium iodide; AV, Annexin V; IL, interleukin, IL2RG, interleukin 2 receptor $\gamma$; ITK-SYK, IL-2-inducible T-cell kinase-spleen tyrosine kinase; JAK, tyrosine-protein kinase JAK; p27, cyclin-dependent kinase inhibitor 1B; CDK2, cyclin-dependent kinase 2; NS, not significant; si-control, scrambled siRNA; si or siRNA, small interfering RNA. *P<0.05 and ** $<<0.01$. 


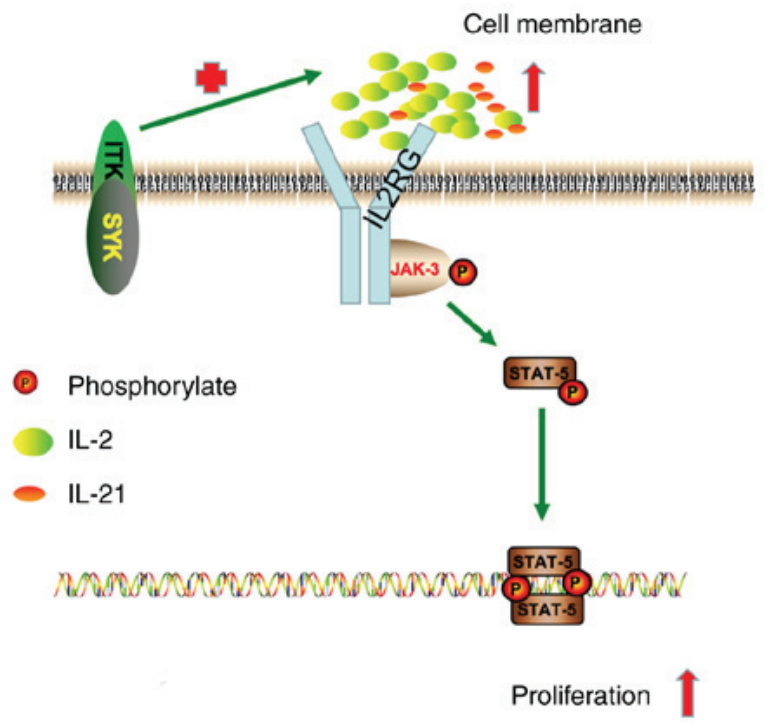

Figure 6. Graphical abstract of the model of the activation of IL2RG/JAK/STAT following the overexpression of the ITK-SYK fusion gene. ITK-SYK overexpression leads to the maximal production of IL-2 and IL-21. The binding of cytokines to IL2RG is increased. The engagement of IL2RG triggers the JAK/STAT signalling pathway. Increased phosphorylated STAT5 expression leads to the promotion of peripheral T-cell lymphoma cel proliferation. ITK-SYK, IL-2-inducible T-cell kinase-spleen tyrosine kinase; JAK, tyrosine-protein kinase JAK; IL, interleukin, IL2RG, interleukin 2 receptor $\gamma$.

anti-tumourigenic potential of tofacitinib on tumour growth was evident after day 13. CEM cells were transduced with a lentiviral construct conferring EGFP expression to enable fluorescence detection. It was found that tofacitinib significantly decreased the radiant efficiency, showing that tumour growth was suppressed (Fig. 4B). Immunohistochemical analysis showed that the immunostaining intensity of SYK was significantly stronger in the control group than in the tofacitinib group (Fig. 4C).

JAK3 phosphorylation was dependent on the expression of $I L 2 R G$, and associated with the secretion of IL-2 and IL-21. JAK3 is a tyrosine kinase that participates in the signalling of several cytokines (IL-2, IL-4, IL-7, IL-9, IL-15 and IL-21) through receptors that share the IL2RG chain (29). To determine whether JAK3 activation shares a similar receptor-dependent mechanism of constitutive signalling in ITK-SYK ${ }^{+}$Jurkat cells, the effects of IL2RG-knockdown on ITK-SYK ${ }^{+}$Jurkat cells was analysed and three IL2RG-specific siRNAs were used to inhibit IL2RG signalling at the gene level. siIL2RG-2 effectively inhibited the level of IL2RG compared with silL2RG-1 and siIL2RG-3 (Fig. 5A). The growth curve showed that IL2RG-knockdown significantly inhibited the number of ITK-SYK ${ }^{+}$Jurkat cells, which was increased in both groups with the treatment duration (24, 48, 72 and $96 \mathrm{~h}$; Fig. 5C). Similarly, IL2RG-knockdown induced a significant increase in ITK-SYK ${ }^{+}$Jurkat cell apoptosis compared with the si-control-treated cells (Fig. 5D). Consistent with the results obtained by flow cytometry, increased levels of cleaved caspase-3, together with decreased levels of full-length caspase-3, were observed in the silL2RG-2-treated group (Fig. 5E). Cell cycle analysis demonstrated a significant increase in the number of cells in the G1/S phase in siIL2RG-2-treated ITK-SYK ${ }^{+}$Jurkat cells (control 76.23\% vs. siIL2RG-2 92.31\%; $\mathrm{P}<0.01$; Fig. 5F). Consistent with the analysis of G1/S phase arrest, increased levels of p27 and decreased levels of CDK2 were observed in ITK-SYK ${ }^{+}$Jurkat cells following treatment with silL2RG-2 (Fig. 5G). The associated between the secretion of cytokines and IL2RG in ITK-SYK ${ }^{+}$Jurkat cells was then investigated. IL-2 and IL-21 production was significantly induced in ITK-SYK ${ }^{+}$Jurkat cells compared with that in ITK-SYK- Jurkat cells, while the secretion of IL-4, IL-7, IL-9 and IL-15 was not significantly affected (Fig. 5H). IL-2 and IL-21 production in IL2RG-knocked down ITK-SYK ${ }^{+}$Jurkat cells was not significantly affected when compared with that in the control. The level of tyrosine phosphorylation of STAT5 is a direct indicator of the presence of a functional IL-2 receptor complex, therefore the levels of tyrosine phosphorylation in STAT5 were measured. The silencing of IL2RG by siIL2RG-2 significantly suppressed the phosphorylation of JAK3 and STAT5 in ITK-SYK ${ }^{+}$Jurkat cells, while si-control did not affect it (Fig. 5B). Meanwhile, extrinsic IL-2 $(25 \mathrm{ng} / \mathrm{ml})$ and IL-21 $(25 \mathrm{ng} / \mathrm{ml})$ did not restore the changes caused by siIL2RG-2. Consistent with the results of the cytokine expression analysis, it was found that the expression levels of IL2RA and IL21R were elevated in ITK-SYK ${ }^{+}$Jurkat cells, while that of IL7R was decreased (Fig. 5I). Taken together, these findings showed that JAK3/STA5 phosphorylation was dependent on the presence of IL2RG in ITK-SYK ${ }^{+}$Jurkat cells, and the overexpression of fusion gene was associated with the secretion of IL-2 and IL-21. All of these data strongly suggest that IL2RG/JAK/STAT activation is involved in PTCLs with the ITK-SYK fusion gene (Fig. 6).

\section{Discussion}

The $\mathrm{t}(5 ; 9)(\mathrm{q} 33 ; \mathrm{q} 22)$ translocation that results in the constitutive activation of ITK-SYK tyrosine kinase is a key oncogenic event in the development of PTCLs (7). In previous studies, ITK-SYK was found to mimic the TCR signalling pathway and activate the downstream PI3K-Akt and MAPK pathways (15-18). However, the exact mechanism underlying ITK-SYK fusion is not well understood. In the current study, mRNA expression profiling found that DEGs between ITK-SYK ${ }^{+}$and ITK-SYK cells were enriched in JAK/STAT, TCR, PI3K and MAPK signalling pathways. Furthermore, the elevated secretion of IL-2 and IL-21 was induced by ITK-SYK. Previous studies have reported that ITK-SYK triggers the profound production of IL-2 in ITK-SYK ${ }^{+}$Jurkat cells at levels even higher $(16,18)$ than those induced by optimal CD3/CD28 co-stimulation in the current study.

Signal-Net analysis with microarray data highlighted the deregulation of the JAK/STAT pathway in ITK-SYK ${ }^{+}$ PTCLs. The JAK/STAT pathway is instrumental for the differentiation, proliferation and survival of a variety of cell types, including those of the haematopoietic lineages (30). The JAK family includes JAK1, JAK2, JAK3 and TYK2 (31). Unlike JAK1, JAK2 and TYK2 are ubiquitously expressed, while JAK3 is restricted in the haematopoietic lineage and plays a vital role in lymphoid cell development and proliferation (32-34). In mouse models, the constitutive activation of JAK3 results in megakaryocyte hyperplasia or 
lymphoproliferative disease $(34,35)$. In human pathology, JAK3 activation was found in haematological malignancies, including adult T-cell leukaemia/lymphoma (36), cutaneous T-cell lymphoma (37,38), natural killer/T-cell lymphoma (39), T-ALL (40), T-cell prolymphocytic leukaemia (41) and acute myeloid leukaemia (42). The majority of abnormal JAK3 activation is related to gain-of-function mutations $(43,44)$. Most JAK3 mutants identified in samples from patients with T-ALL were capable of conferring cytokine-independent growth to cell lines in vitro and causing leukaemia in vivo $(43,45)$. However, in certain extranodal NK/T-cell lymphoma nasal type cases reported in Asia, the function of JAK3 was altered regardless of their mutation status, mainly due to phosphorylation $(46,47)$.

In the current study, JAK3 protein levels were moderately increased, while the phosphorylation of JAK3 was constitutively activated in ITK-SYK ${ }^{+}$Jurkat cells. IL2RG is essential to JAK3 activation (48). In the present study, silencing IL2RG resulted in the inactivation of JAK3 and downstream STAT5. Similar to the results of the current study, Fathi et al (10) also observed that ITK-SYK significantly enhanced phosphorylation of STAT. However, the ITK-SYK fusion gene activated STAT3 instead of STAT5 in their study, possibly due to the use of different cell lines (10). Sprissler et al (49) have reported that depletion of STAT5 blocks SYK-induced transformation. Their study also used a SYK fusion gene, but they investigated the downstream mediator of TEL-SYK instead of ITK-SYK (49). Several studies have suggested that JAK1 is an essential kinase required downstream of activated JAK3 $(43,50)$. Indeed, a close relationship exists between JAK1 and JAK 3 at the normal IL-7 receptor, where both kinases phosphorylate and activate each other upon stimulation of the receptor (43). However, in the present study, ITK-SYK caused the phosphorylation of JAK3, but not JAK1. Furthermore, IL-7R was downregulated by ITK-SYK, consistent with its JAK1-inactivated status. Taken together, these results suggest that JAK3 activation by ITK-SYK was independent of JAK1. Further experiments are required to explore the mechanisms underlying the activation of JAK3 by the fusion gene.

Considering the restricted expression pattern and function of JAK3 in lymphoid tissues, JAK3 appears to be a possible target for therapy in various haematologic malignancies. In the present study, the anti-tumour effects of the JAK3 inhibitor tofacitinib against ITK-SYK ${ }^{+}$Jurkat cells were evaluated in vitro and in a murine xenograft model. Tofacitinib suppressed cell growth, and induced apoptosis and G1/S phase arrest. In the xenograft model, subcutaneous inoculation of ITK-SYK ${ }^{+}$CEM cells into NOD/SCID mice resulted in tumour formation, and tofacitinib markedly suppressed tumour growth. As tofacitinib is currently being assessed in the clinic for the treatment of inflammatory diseases, it could also be assessed in T-cell lymphoma in clinical trials. In previous studies, Streubel et al (6) detected five PTCL cases with the ITK-SYK fusion gene in 30 patients with unspecified PTCL. However, Feldman et al (51) could not detect the fusion gene in 141 patients with PTCL. The authors of the current study attempted to detect the clinical cases of PTCLs with the ITK-SYK fusion gene. However, only one case with the fusion gene was detected in fresh tissues by reverse transcription-quantitative PCR, therefore it was not mentioned in the current study.
In conclusion, the authors of the current study demonstrated that the IL2RG/JAK3/STAT5 signalling pathway was activated in ITK-SYK ${ }^{+}$Jurkat cells, and this activation was associated with the abundant secretion of IL-2 and IL-21 driven by the fusion gene. Finally, the authors of the current study determined that the JAK3-specific inhibitor tofacitinib shows potential as a new therapeutic agent against PTCLs with the ITK-SYK fusion gene. Further studies should focus on the relationship between ITK-SYK ${ }^{+}$tumour cells and T follicular helper cells.

\section{Acknowledgements}

Not applicable.

\section{Funding}

The current study was supported by the National Natural Science Foundation of China (grant no. 81400163).

\section{Availability of data and materials}

All data generated or analysed during the present study are included in the published article.

\section{Authors' contributions}

LLZ, LL and TG designed the study and participated in drafting the manuscript. LLZ, HXP and YXW performed the experiments. All authors were responsible for data collection and analysis, and read and approved the final version of the manuscript.

\section{Ethics approval and consent to participate}

The current study was approved on $01 / 16 / 2017$ by the Institutional Animal Care and Use Committee at Tongji Medical College, Huazhong University of Science and Technology (Wuhan, China; ACUC No. S842).

\section{Patient consent for publication}

Not applicable.

\section{Competing interests}

The authors declare no competing financial interests.

\section{References}

1. Broccoli A and Zinzani PL: Peripheral T-cell lymphoma, not otherwise specified. Blood 129: 1103-1112, 2017.

2. Said J and Pinter-Brown L: Clinical and pathological diagnosis of peripheral T-cell lymphoma and emerging treatment options: A case-based discussion. Clin Adv Hematol Oncol 7 (Suppl): S1, S4-13, S15, 2009.

3. Bisig B, Gaulard P and de Leval L: New biomarkers in T-cell lymphomas. Best Pract Res Clin Haematol 25: 13-28, 2012.

4. Boddicker RL, Razidlo GL, Dasari S,Zeng Y, Hu G, Knudson RA, Greipp PT, Davila JI, Johnson SH, Porcher JC, et al: Integrated mate-pair and RNA sequencing identifies novel, targetable gene fusions in peripheral T-cell lymphoma. Blood 128: 1234-1245, 2016. 
5. Sandell RF, Boddicker RL and Feldman AL: Genetic landscape and classification of peripheral $\mathrm{T}$ cell lymphomas. Curr Oncol Rep 19: 28, 2017.

6. Streubel B, Vinatzer U, Willheim M, Raderer M and Chott A: Novel $\mathrm{t}(5 ; 9)(\mathrm{q} 33 ; \mathrm{q} 22)$ fuses ITK to SYK in unspecified peripheral T-cell lymphoma. Leukemia 20: 313-318, 2006.

7. Bach MP, Hug E, Werner M, Holch J, Sprissler C, Pechloff K, Zirlik K, Zeiser R, Dierks C, Ruland J and Jumaa H: Premature terminal differentiation protects from deregulated lymphocyte activation by ITK-Syk. J Immunol 192: 1024-1033, 2014.

8. Huang Y, Moreau A, Dupuis J, Streubel B, Petit B, Le Gouill S, Martin-Garcia N, Copie-Bergman C, Gaillard F, Qubaja M, et al: Peripheral T-cell lymphomas with a follicular growth pattern are derived from follicular helper T cells (TFH) and may show overlapping features with angioimmunoblastic T-cell lymphomas. Am J Surg Pathol 33: 682-690, 2009.

9. Attygalle AD, Feldman AL and Dogan A: ITK/SYK translocation in angioimmunoblastic T-cell lymphoma. Am J Surg Pathol 37: 1456-1457, 2013.

10. Fathi NN, Mohammad DK, Görgens A, Andaloussi SE, Zain R, Nore BF and Smith CIE: Translocation-generated ITK-FER and ITK-SYK fusions induce STAT3 phosphorylation and CD69 expression. Biochem Biophys Res Commun 504: 749-752, 2018

11. Andreotti AH, Schwartzberg PL, Joseph RE and Berg LJ: T-cell signaling regulated by the Tec family kinase, Itk. Cold Spring Harb Perspect Biol 2: a002287, 2010.

12. Prince AL, Yin CC, Enos ME, Felices M and Berg LJ: The Tec kinases Itk and Rlk regulate conventional versus innate T-cell development. Immunol Rev 228: 115-131, 2009.

13. Rickert RC: New insights into pre-BCR and BCR signalling with relevance to B cell malignancies. Nat Rev Immunol 13: 578-591, 2013.

14. Tsang E, Giannetti AM, Shaw D, Dinh M, Tse JK, Gandhi S, Ho H, Wang S, Papp E and Bradshaw JM: Molecular mechanism of the Syk activation switch. J Biol Chem 283: 32650-32659, 2008.

15. Hussain A, Faryal R, Nore BF, Mohamed AJ and Smith CI: Phosphatidylinositol-3-kinase-dependent phosphorylation of SLP-76 by the lymphoma-associated ITK-SYK fusion-protein. Biochem Biophys Res Commun 390: 892-896, 2009.

16. Hussain A, Mohammad DK, Gustafsson MO, Uslu M, Hamasy A, Nore BF, Mohamed AJ and Smith CI: Signaling of the ITK (IL2-inducible T-cell kinase)-SYK fusion kinase is dependent on adapter SLP-76 (SH2 domain-containing leukocyte protein of $76 \mathrm{kD}$ ) and on the adapter function of the kinases SYK/ZAP70 (zeta-chain [TCR] associated protein kinase $70 \mathrm{kD}$ ). J Biol Chem 288: 7338-7350, 2013.

17. Dierks C, Adrian F, Fisch P, Ma H, Maurer H, Herchenbach D, Forster CU, Sprissler C, Liu G, Rottmann S, et al: The ITK-SYK fusion oncogene induces a T-cell lymphoproliferative disease in mice mimicking human disease. Cancer Res 70: 6193-6204, 2010.

18. Pechloff K, Holch J, Ferch U, Schweneker M, Brunner K, Kremer M, Sparwasser T, Quintanilla-Martinez L, Zimber-Strobl U, Streubel B, et al: The fusion kinase ITK-SYK mimics a $\mathrm{T}$ cell receptor signal and drives oncogenesis in conditional mouse models of peripheral T cell lymphoma. J Exp Med 207: 1031-1044, 2010.

19. Kanehisa M and Goto S: KEGG: Kyoto encyclopedia of genes and genomes. Nucleic Acids Res 28: 27-30, 2000.

20. Kanehisa M, Sato Y, Kawashima M, Furumichi M and Tanabe M: KEGG as a reference resource for gene and protein annotation. Nucleic Acids Res 44: D457-D462, 2016.

21. Huang da W, Sherman BT and Lempicki RA: Bioinformatics enrichment tools: Paths toward the comprehensive functional analysis of large gene lists. Nucleic Acids Res 37: 1-13, 2009.

22. Huang da W, Sherman BT and Lempicki RA: Systematic and integrative analysis of large gene lists using DAVID bioinformatics resources. Nat Protoc 4: 44-57, 2009.

23. Shannon P, Markiel A, Ozier O, Baliga NS, Wang JT, Ramage D, Amin N, Schwikowski B and Ideker T: Cytoscape: A software environment for integrated models of biomolecular interaction networks. Genome Res 13: 2498-2504, 2003.

24. Chen KT, Hour MJ, Tsai SC, Chung JG, Kuo SC, Lu CC, Chiu YJ, Chuang YH and Yang JS: The novel synthesized 6-fluoro(3-fluorophenyl)-4-(3-methoxyanilino)quinazoline (LJJ-10) compound exhibits anti-metastatic effects in human osteosarcoma U-2 OS cells through targeting insulin-like growth factor-I receptor. Int J Oncol 39: 611-619, 2011
25. Liao CL, Lai KC, Huang AC, Yang JS, Lin JJ, Wu SH, Gibson Wood W, Lin JG and Chung JG: Gallic acid inhibits migration and invasion in human osteosarcoma U-2 OS cells through suppressing the matrix metalloproteinase-2/-9, protein kinase $\mathrm{B}$ (PKB) and PKC signaling pathways. Food Chem Toxicol 50: 1734-1740, 2012.

26. Khokhlova ON, Tukhovskaya EA, Kravchenko IN, Sadovnikova ES, Pakhomova IA, Kalabina EA, Lobanov AV, Shaykhutdinova ER, Ismailova AM and Murashev AN: Using Tiletamine-Zolazepam-Xylazine anesthesia compared to $\mathrm{CO}_{2}$-inhalation for terminal clinical chemistry, hematology, and coagulation analysis in mice. J Pharmacol Toxicol Methods 84: $11-19,2017$.

27. Rigby S, Huang Y, Streubel B, Chott A, Du MQ, Turner SD and Bacon CM: The lymphoma-associated fusion tyrosine kinase ITK-SYK requires pleckstrin homology domain-mediated membrane localization for activation and cellular transformation. J Biol Chem 284: 26871-26881, 2009.

28. Eißmann M, Melzer IM, Fernandez SB, Michel G, Hrabě de Angelis M, Hoefler G, Finkenwirth P, Jauch A, Schoell B, Grez M, et al: Overexpression of the anti-apoptotic protein AVEN contributes to increased malignancy in hematopoietic neoplasms. Oncogene 32: 2586-2591, 2013.

29. Hekmatnejad M, Conwell S, Lok SM, Kutach A, Shaw D, Fang E and Swinney DC: Insights into kinetic mechanism of Janus kinase 3 and its inhibition by tofacitinib. Arch Biochem Biophys 612: 22-34, 2016.

30. O'Shea JJ, Schwartz DM, Villarino AV, Gadina M, McInnes IB and Laurence A: The JAK-STAT pathway: Impact on human disease and therapeutic intervention. Annu Rev Med 66: 311-328, 2015.

31. Goll GL and Kvien TK: New-generation JAK inhibitors: How selective can they be? Lancet 391: 2477-2478, 2018.

32. Ross JA, Spadaro M, Rosado DC, Cavallo F, Kirken RA and Pericle F: Inhibition of JAK3 with a novel, selective and orally active small molecule induces therapeutic response in T-cell malignancies. Leukemia 28: 941-944, 2014.

33. Kwatra SG: The role of Jak3 signaling in IL-17 expression in malignant cutaneous T-cell lymphoma. J Invest Dermatol 131: 1954-1956, 2011

34. Cornejo MG, Kharas MG, Werneck MB, Le Bras S, Moore SA, Ball B, Beylot-Barry M, Rodig SJ, Aster JC, Lee BH, et al: Constitutive JAK3 activation induces lymphoproliferative syndromes in murine bone marrow transplantation models. Blood 113: 2746-2754, 2009.

35. Cornejo MG, Boggon TJ and Mercher T: JAK3: A two-faced player in hematological disorders. Int J Biochem Cell Biol 41: 2376-2379, 2009.

36. Tomita M, Kawakami H, Uchihara JN, Okudaira T, Masuda M, Matsuda T, Tanaka Y, Ohshiro K and Mori N: Inhibition of constitutively active Jak-Stat pathway suppresses cell growth of human T-cell leukemia virus type 1-infected T-cell lines and primary adult T-cell leukemia cells. Retrovirology 3: 22 , 2006.

37. Krejsgaard T, Vetter-Kauczok CS, Woetmann A, Lovato $P$, Labuda T, Eriksen KW, Zhang Q, Becker JC and Ødum N: Jak3- and JNK-dependent vascular endothelial growth factor expression in cutaneous T-cell lymphoma. Leukemia 20: $1759-1766,2006$

38. Lauenborg B, Christensen L, Ralfkiaer U, Kopp KL, Jønson L, Dabelsteen S, Bonefeld CM, Geisler C, Gjerdrum LM, Zhang $\mathrm{Q}$, et al: Malignant $\mathrm{T}$ cells express lymphotoxin $\alpha$ and drive endothelial activation in cutaneous $\mathrm{T}$ cell lymphoma. Oncotarget 6: 15235-15249, 2015.

39. Yan J, Li B, Lin B, Lee PT, Chung TH, Tan J, Bi C, Lee XT, Selvarajan V, Ng SB, et al: EZH2 phosphorylation by JAK3 mediates a switch to noncanonical function in natural killer/T-cell lymphoma. Blood 128: 948-958, 2016.

40. Neumann M, Vosberg S, Schlee C, Heesch S, Schwartz S, Gökbuget N, Hoelzer D, Graf A, Krebs S, Bartram I, et al: Mutational spectrum of adult T-ALL. Oncotarget 6: 2754-2766, 2015.

41. Stengel A, Kern W, Zenger M, Perglerová K, Schnittger S, Haferlach T and Haferlach C: Genetic characterization of T-PLL reveals two major biologic subgroups and JAK3 mutations as prognostic marker. Genes Chromosomes Cancer 55: 82-94, 2016.

42. Wu AY, Yang HC, Lin CM, Wu BD, Qu QS, Zheng YH, Wei H, Mei XQ, Zeng ZH and Ma XD: The transcriptome study of subtype M2 acute myeloblastic leukemia. Cell Biochem Biophys 72: 653-656, 2015. 
43. Degryse S, de Bock CE, Cox L, Demeyer S, Gielen O, Mentens N, Jacobs K, Geerdens E, Gianfelici V, Hulselmans G, et al: JAK3 mutants transform hematopoietic cells through JAK1 activation, causing T-cell acute lymphoblastic leukemia in a mouse model. Blood 124: 3092-3100, 2014.

44. Jeong EG, Kim MS, Nam HK, Min CK, Lee S, Chung YJ, Yoo NJ and Lee SH: Somatic mutations of JAK1 and JAK3 in acute leukemias and solid cancers. Clin Cancer Res 14: 3716-3721, 2008.

45. Waldmann TA and Chen J: Disorders of the JAK/STAT pathway in $\mathrm{T}$ cell lymphoma pathogenesis: Implications for immunotherapy. Annu Rev Immunol 35: 533-550, 2017.

46. Kimura H, Karube K, Ito Y, Hirano K, Suzuki M, Iwata S and Seto M: Rare occurrence of JAK3 mutations in natural killer cell neoplasms in Japan. Leuk Lymphoma 55: 962-963, 2014.

47. Lee S, Park HY, Kang SY, Kim SJ, Hwang J, Lee S, Kwak SH, Park KS, Yoo HY, Kim WS, et al: Genetic alterations of JAK/STAT cascade and histone modification in extranodal NK/T-cell lymphoma nasal type. Oncotarget 6: 17764-17776, 2015.

48. Lindemann MJ, Benczik M and Gaffen SL: Anti-apoptotic signaling by the interleukin-2 receptor reveals a function for cytoplasmic tyrosine residues within the common gamma (gamma c) receptor subunit. J Biol Chem 278: 10239-10249, 2003.
49. Sprissler C, Belenki D, Maurer H, Aumann K, Pfeifer D, Klein C, Müller TA, Kissel S, Hülsdünker J, Alexandrovski J, et al: Depletion of STAT5 blocks TEL-SYK-induced APMF-type leukemia with myelofibrosis and myelodysplasia in mice. Blood Cancer J 4: e240, 2014.

50. Kiel MJ, Velusamy T, Rolland D, Sahasrabuddhe AA, Chung F, Bailey NG, Schrader A, Li B, Li JZ, Ozel AB, et al: Integrated genomic sequencing reveals mutational landscape of T-cell prolymphocytic leukemia. Blood 124: 1460-1472, 2014.

51. Feldman AL, Sun DX, Law ME, Novak AJ, Attygalle AD, Thorland EC, Fink SR, Vrana JA, Caron BL, Morice WG, et al: Overexpression of Syk tyrosine kinase in peripheral T-cell lymphomas. Leukemia 22: 1139-1143, 2008.

(i) $\Theta$ This work is licensed under a Creative Commons Attribution-NonCommercial-NoDerivatives 4.0 International (CC BY-NC-ND 4.0) License. 